Check for updates

Cite this: Mol. Omics, 2018, 14,134

DOI: $10.1039 / c 8 m o 90005 a$

rsc.li/molomics

\section{Correction: Selenium effects on the metabolism of a Se-metabolizing Lactobacillus reuteri: analysis of envelope-enriched and extracellular proteomes}

\author{
E. Pessione, ${ }^{\star a}$ C. Lamberti, ${ }^{\text {b }}$ A. Pessione, ${ }^{a}$ E. Galano, ${ }^{c}$ A. Amoresano ${ }^{c}$ and \\ E. Mangiapane ${ }^{a}$
}

Correction for 'Selenium effects on the metabolism of a Se-metabolizing Lactobacillus reuteri: analysis of envelope-enriched and extracellular proteomes' by E. Mangiapane et al., Mol. BioSyst., 2014, 10, 1272-1280.

Regrettably, in the original article, there is an error in the reported name of the bacterial strain. The bacterial strain was reported as Lactobacillus reuteri Lb2 (DSM 16143) but it should have been reported as Lactobacillus reuteri Lb26 (DSM 16341). Therefore, any instances of Lb2 (DSM 16143) should be replaced with Lb26 (DSM 16341).

The experimental work was conducted on the strain Lactobacillus reuteri Lb26 (DSM 16341), formerly classified as Lactobacillus buchneri Lb26 (DSM 16341). Unfortunately the strain provided was mislabeled as Lactobacillus reuteri Lb2 (DSM 16143), which does not possess any of the specific traits published.

The results of the research were unaffected by this labelling issue.

The Royal Society of Chemistry apologises for these errors and any consequent inconvenience to authors and readers.

\footnotetext{
${ }^{a}$ Department of Life Sciences and Systems Biology, University of Turin, Via Accademia Albertina 13, 10123, Torino, Italy. E-mail: enrica.pessione@unito.it

${ }^{b}$ CNR ISPA, c/o Bioindustry Park S. Fumero, Via Ribes 5, 10010, Colleretto Giacosa, Italy

'Department of Chemical Sciences, University of Naples "Federico II", Via Cinthia 4, 80125, Napoli, Italy
} 